\title{
Dynamics of multifractal and correlation characteristics of the spatio-temporal distribution of regional seismicity before the strong earthquakes
}

\author{
D. Kiyashchenko ${ }^{1}$, N. Smirnova ${ }^{1}$, V. $\operatorname{Troyan}^{1}$, and F. Vallianatos ${ }^{2}$ \\ ${ }^{1}$ Institute of Physics, St. Petersburg University, St. Petersburg, 198504, Russia \\ ${ }^{2}$ Technological Educational Institute of Crete, Chania Crete, Greece
}

Received: 3 July 2002 - Revised: 25 February 2003 - Accepted: 26 February 2003

\begin{abstract}
Investigations of the distribution of regional seismicity and the results of numerical simulations of the seismic process show the increase of inhomogenity in spatiotemporal distribution of the seismicity prior to large earthquakes and formation of inhomogeneous clusters in a wide range of scales. Since that, the multifractal approach is appropriate to investigate the details of such dynamics.

Here we analyze the dynamics of the seismicity distribution before a number of strong earthquakes occurred in two seismically active regions of the world: Japan and Southern California. In order to study the evolution of spatial inhomogeneity of the seismicity distribution, we consider variations of two multifractal characteristics: information entropy of multifractal measure generation process and the higher-order generalized fractal dimension of the continuum of the earthquake epicenters. Also we studied the dynamics of the level of spatio-temporal correlations in the seismicity distribution. It is found that two aforementioned multifractal characteristics tend to decrease and the level of spatio-temporal correlations tends to increase before the majority of considered strong earthquakes. Such a tendency can be considered as an earthquake precursory signature.

Therefore, the results obtained show the possibility to use multifractal and correlation characteristics of the spatiotemporal distribution of regional seismicity for seismic hazard risk evaluation.
\end{abstract}

\section{Introduction}

Study of evolution of spatial and temporal distribution of the seismicity is important for understanding of the earthquake preparation process. A number of precursory phenomena is related to changes in spatial distribution of the seismicity. One of the common features of these phenomena is the evolution of inhomogeneity in the seismicity spatial distri-

Correspondence to: D. Kiyashchenko

(denis@geo.phys.spbu.ru) bution in wide range of scales. In the large scales, this feature manifests itself in the seismicity concentration close to the nodal plane of the future rupture. The laboratory experiments of Mogi and Scholz (Mogi, 1968; Scholz, 1968) have to be mentioned in this connection. Also this pattern has been observed in the laboratory experiments of Sobolev and Ponomarev (1999) and even in the actual studies of the seismicity behavior before some strong earthquakes of the Kamchatka region (Zavialov and Nikitin, 1999). In the small scales, this phenomena manifests itself in the seismicity clustering before the mainshock (Zavialov and Nikitin, 1999) and formation of spatio-temporal clusters of the acoustic emission in the laboratory experiments (Sobolev and Ponomarev, 1999).

A suitable method for description of the inhomogeneity of the seismicity spatial distribution is based on fractal approach. The results of corresponding investigations show that seismicity spatial distribution manifests statistically selfsimilar properties in a wide range of scales. Therefore, it can be treated as fractal or multifractal. Several papers are related to this item (Sadovsky et al., 1984; Okubo and Aki, 1987; Geilikman et. al., 1990; Hirata and Imoto, 1991; Turcotte, 1997; Wang and Lee, 1996; Lapenna et al., 2000). However, only a few papers are reported, where the dynamics of fractal properties of seismicity is studied before strong earthquakes.

A gradual decrease of the correlation exponent of spatial distribution of acoustic shocks has been observed by Hirata et al. (1987) during the destruction of granite sample. The behavior of the fractal dimension (calculated by the boxcounting method) of the continuum of earthquake epicenters has been studied by Uritsky and Troyan (1998). The author used the materials of the world-wide seismicity data-center. The fractal dimension had been calculated for the two-year periods before and after 23 strong earthquakes. In 16 cases the pre-earthquake period was characterized by a lower fractal dimension.

The results of numerical modeling of the destruction process of the elastic body, containing a number of cracks (Kiyashchenko and Troyan, 2001), showed that correlation dimension of the continuum of the synthetic seismic- 

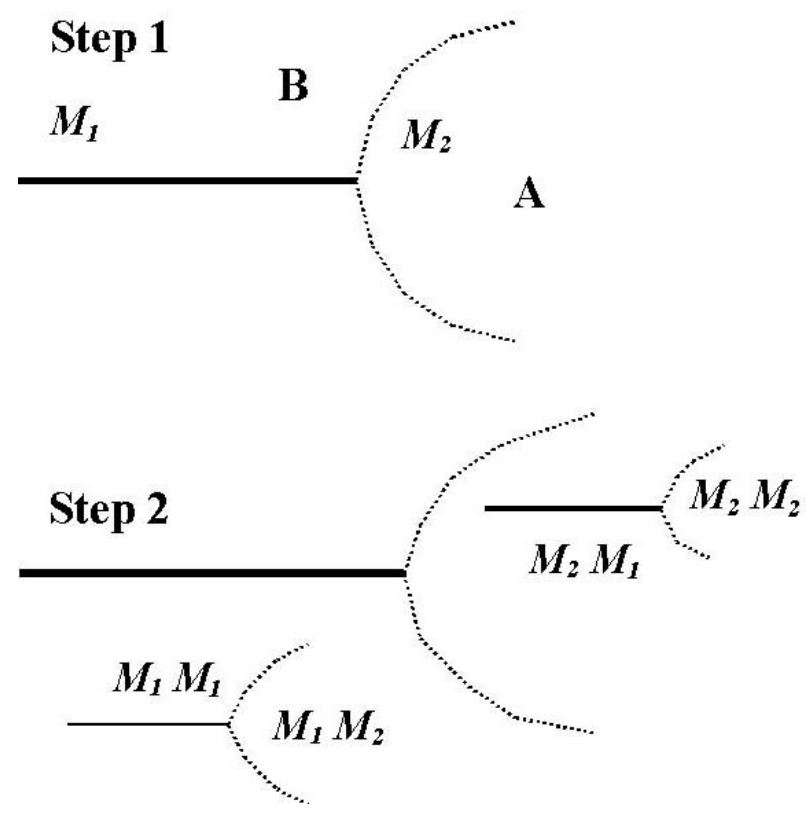

Fig. 1. Qualitative analogy between destruction process and multiplicative process.

ity hypocenters decreased during evolution of the destruction process towards the main rupture. Hence, evolution of the inhomogeneity of spatial distribution of seismicity in different scales, which manifests itself in decrease of fractal dimensions, can be interpreted as a significant feature of the earthquake preparation process.

In this paper we apply multifractal methods for analysis of the seismicity distribution dynamics prior to strong earthquakes. There are several reasons for using multifractal analysis instead of monofractal analysis (i.e. calculation of fractal dimension by box-counting method, calculation of correlation dimension, etc.):

1. Multifractal analysis is more general than fractal analysis. Fractal dimension and correlation dimension can be easily retrieved from the spectrum of the generalized fractal dimensions.

2. Fractal analysis, based on the box-counting algorithm (i.e. counting of the number of non-empty boxes of different sizes, covering the considered continuum), can not take into account the distribution of number of earthquakes along different boxes. Multifractal analysis fills this gap of fractal analysis, since it is based on counting of the number of events in the boxes of different sizes.

If we represent the seismicity distribution as a colour map, the fractal analysis treats it as a black/white image. Multifractal analysis takes into account different colors, which compose the image.

3. The qualitative analogy can be established between the destruction process and multiplicative process of multifractal structure generation. The crack, appearing in the elastic body under external stress (Fig. 1, Step 1),

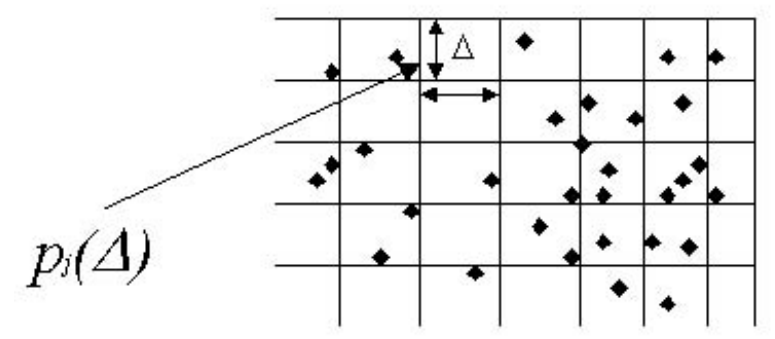

Fig. 2. Earthquake epicenters continuum.

causes redistribution of stress near it's tips (see Lawn and Wilshaw, 1977; Liebowits, 1968). The notions $M_{2}$, $M_{1}\left(M_{2}>M_{1}\right)$ in Fig. 1 are relative values of average stress level in the zones $B, A$, respectively. The cracks, appearing in the zones $A, B$ cause the stress redistribution in similar proportion (Step 2). As a result of a number of such steps, the highly inhomogeneous spotlike stress field distribution and hierarchical crack system appear in the elastic body. The spot-like feature of the seismicity can be considered as one of the manifestations of such mechanism.

Therefore, multifractal methods seems to be suitable for description of the seismicity distribution.

Besides study of precursory evolution of inhomogenity of spatial distribution of the seismicity, it is also important to pay attention to the evolution of inhomogenity in the temporal distribution of the seismicity. The results of numerical modeling of the destruction process (Kiyashchenko and Troyan, 2001) and the results of study of the acoustic emission (Sobolev and Ponomarev, 1999) show the increase of spatio-temporal correlations in the seismicity distribution before the main rupture.

Here we study the dynamics of multifractal and spatiotemporal correlation characteristics of the seismicity before several strong earthquakes of Japan and Southern California in order to reveal precursory signatures.

\section{The approach to study of the seismicity distribution dynamics}

In this section we give the description of our approach to study of the dynamics of spatio-temporal distribution of the seismicity prior to large earthquakes.

We use multifractal analysis for description of spatial distribution of seismicity. As it is discussed in the previous section, the multifractal approach is suitable for this purpose. Moreover, the variations of scaling characteristics of the seismicity have to contain information about the evolution of the destruction process in the earth crust (see Kiyashchenko and Troyan, 2001).

The detailed description of multifractal formalism can be found in Feder, 1988 and Mandelbrot, 1989. The brief de- 
scription of principal points of multifractal analysis is given below in Appendix A.

As it is shown in the previous section, the spatial distribution of the seismicity can be treated as a result of some cascade multiplicative process, governed by a set of parameters. The binomial multiplicative process, which is governed by two parameters only, is the simplest one among such kinds of processes. It is considered in Appendix A.

The distributions, which are generated by multiplicative processes, have the properties of multifractals. Such distributions can be completely described by the spectrum of generalized fractal dimensions $d(q)$ or the multifractal spectrum $f(\alpha)$ (see Appendix A). These characteristics contain a lot of information about the parameters of multiplicative process, underlying the generation of multifractal distribution.

In our case, we study 2D distribution of the seismicity hypocenters (Fig. 2). The distribution function $p_{j}(\Delta)$ is defined as:

$p_{j}(\Delta)=\frac{N_{j}}{N}$,

where $N_{j}$ is the number of earthquakes occurred in the cell with number $j$ and size $\Delta$, and $N$ is the total number of earthquakes in the considered region. The generalized fractal dimension $d(q)$ of the order $q$ is determined as a slope of the best fit line representing the Renyi entropy $I_{q}(\Delta)$ versus $1 / \Delta$ in log-log plot (see Eqs. (A3) and (A4) in Appendix A).

The dynamics of two multifractal characteristics have been studied prior to strong earthquakes: the minimal value of the Holder exponent $\alpha_{\min }=d(\infty)$ and the entropy $S$ of the multifractal measure generation process $\left(S=\alpha_{1}=d(1)\right)$. This multifractal characteristics provide an information about the inhomogeneity of the seismicity distribution and the level of seismicity clustering in some range of scales. So, the entropy $S$ can be treated as the measure of inhomogeneity of distribution of the seismicity: the lower (higher) values of $S$ correspond to more (less) inhomogeneous distributions (see Appendix A). The value of $\alpha_{\min }$ characterizes the degree of seismicity clustering in the most seismically active parts of the considered region in some range of scales. The smaller values of $\alpha_{\min }$ can be interpreted as a manifestation of stronger clustering.

Besides multifractal characteristics, the dynamics of the degree of spatio-temporal correlations in the seismicity distribution has been studied as well. The measure of the level of spatio-temporal correlations can be defined as:

$g(a, b, \tau)=\frac{2}{N(N-1)} N_{p}(\tau, a<R<b) ; \tau=\frac{T_{0}}{W N}$.

Here $N$ is the number of events, occurred in some area during the temporal period $T_{0} ; N_{p}$ is the number of events, occurred within the temporal interval $\tau$, and the spatial distance $R$ between which is ranged from $a$ to $b$. The temporal interval $\tau$ is taken $W$ (in our case $W=50$ ) times less than interval $T_{0} / N$. In this case the function $g(a, b)$ depends only on the temporal distribution of earthquakes, but it does not depend on their total number, occurred during the temporal period $T_{0}$.

The results of study of the dynamics of value $C=$ $\ln (g(0,20 \mathrm{~km}))$ prior to strong earthquakes are presented and discussed in the next section.

Overall, our study contains investigation of the dynamics of the multifractal characteristics $S$ and $\alpha_{\min }$ of spatial distribution of the seismicity and the level $C$ of spatio-temporal correlations in the seismicity prior to several strong earthquakes.

Two earthquake hypocenter catalogs have been used for this analysis:

1. Japan University Network Earthquake Catalog Hypocenters File, published by Tokyo Earthquake Research Institute (http://www.eri.u-tokyo.ac.jp). This catalog contains the earthquakes with magnitude $M>2.0$ occurred in the area with geographical coordinates $\varphi=26-48^{\circ} \mathrm{N}$ and $\lambda=128-148^{\circ} \mathrm{E}$ during the temporal period from 1985 to 1996.

2. Southern California earthquake hypocenter catalog (Hauksson, 2000), containing the earthquakes with magnitude $M>1.0$ occurred in the area with geographical coordinates $\varphi=1-60^{\circ} \mathrm{N}$ and $\lambda=26-178^{\circ} \mathrm{W}$ during the temporal period from 1982 to 1999 (http: //www.scecdc.scec.org/catalog).

We restrict our consideration only with those events, which occurred at the depth less than $60 \mathrm{~km}$. At the large depths the material of the earth crust loses elasticity and the mechanisms of the deep earthquakes could be linked to phase transitions rather than to shear fracturing. The simulation of the destruction of elastic body with a number of shear fractures (see Kiyashchenko and Troyan, 2001) showed the certain dynamics of scaling characteristics and the level of spatio-temporal correlations in the synthetic seismicity prior to main rupture (see Sect. 1). We can expect the similar behavior in the real seismicity, if it is produced by elastic phenomena. That is why we do not consider the deep events occurred due to non-elastic phenomena.

We have selected the strong earthquakes with magnitude $M>6.5$ from both catalogs to study the dynamics of regional seismicity distribution prior to those strong events. For each of the strong earthquakes considered the subcatalog of the seismicity registered in its surrounding area, is selected for analysis. The surrounding area is taken in the form of a box with the side $A$ (Fig. 3) centered at the earthquake epicenter. The size $A$ of the area have to be selected according to the size of the rupture area of the strong earthquake. Also this area should contain sufficient amount of data for multifractal analysis of the seismicity in sliding temporal window.

Let us estimate roughly the available size A of the surrounding area. The size $L$ of the rupture area of the earthquake can be estimated using the empirical relation (Sobolev and Ponomarev, 1999; Sobolev, 1990):

$\lg L=0.6 M-2.5$, 


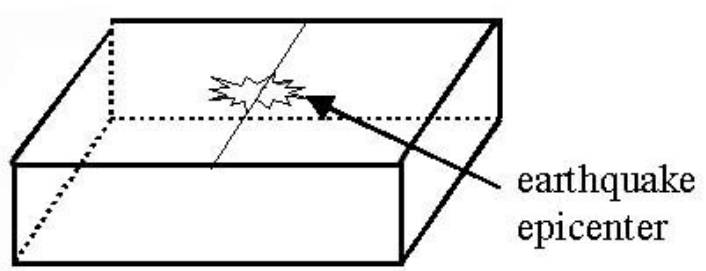

Fig. 3. Box - like area near earthquake epicenter.

Table 1. The rupture size $L$ of the earthquake with magnitude $M$

\begin{tabular}{lccccccccc}
\hline$M$ & 4.0 & 4.5 & 5.0 & 5.5 & 6.0 & 6.5 & 7.0 & 7.5 & 8.0 \\
\hline$L, \mathrm{~km}$ & 0.8 & 1.6 & 3.16 & 6.3 & 12.3 & 25.1 & 50.1 & 100 & 200 \\
\hline
\end{tabular}

where $M$ is the earthquake magnitude.

The values of the rupture size $L$ calculated using Eq. (1), are shown in Table 1.

The effective radius of the surrounding area (equal to $A / 2$ ), which has to be selected for analysis, must not exceed several lengths of the rupture of the expected strong earthquake. That is an important condition for searching of the precursory phenomena using the methods suggested in this paper. In fact, the results of simulation of the destruction of elastic body with a number of cracks (Kiyashchenko and Troyan, 2001) indicate that such precursory tendencies as the decrease of correlation dimension or increase of the level of spatio-temporal correlation in the synthetic seismicity appeared due to interaction of growing cracks in wide range of scales.

In the case of elastic body with two cracks, the interaction between cracks is significant, if the distance between the cracks is comparable with their lengths, and it is negligible, if that distance exceeds several lengths of the larger crack (see Kiyaschchenko and Troyan, 2001). Therefore, if the size of the rupture area of the selected strong earthquake is much smaller than the size of surrounding area (for example if the size of surrounding area is $A=400 \mathrm{~km}$ and the size of rupture area is $L=6.3 \mathrm{~km}$ for the earthquake with $M=5.5$ ), the main part of the seismicity located in this area has no influence on the process of preparation of that strong earthquake.

Since we select the earthquakes with $M>6.5$, the corresponding rupture area $L>25 \mathrm{~km}$ (see Table 1). In such a case the size of the surrounding area $A=200-400 \mathrm{~km}$ seems to be suitable for multifractal analysis: on the one hand, it is in appropriate relation with the rupture size, on the other hand, such area contains sufficient amount of data for analysis of multifractal characteristics of seismicity distribution in sliding temporal windows of reasonable lengths.

Taking into account the amount of data in the sub-catalogs used for analysis, and the chosen temporal window (less than approximately 3 years in our case), the size of surrounding area $400 \times 400 \mathrm{~km}^{2}$ seems to be acceptable for Japanese earthquakes, and the size $200 \times 200 \mathrm{~km}^{2}$ is suitable for the earthquakes of Southern California. We can take the smaller size of surrounding area for the earthquakes of Southern California since the Californian catalogs contain larger amount of data.

Below we present the results of application of our methodology.

\section{The results of study of the dynamics of seismicity dis- tribution prior to strong earthquakes}

Overall, 12 strong earthquakes with $M>6.5$ has been selected, which satisfy the conditions discussed in the previous section. The corresponding sub-catalogs of seismicity in their surrounding areas has been analyzed. The information concerning these earthquakes is summarized in Table 2.

For every of the sub-catalogs the multifractal characteristics ( $S$ and $\alpha_{\min }$ ) and the level of spatio-temporal correlations $C$ are calculated in sliding temporal window. The length of the temporal window $0.3 \times 10^{8} \mathrm{~s}$ (approximately 1 year) is chosen for study of the dynamics of the level of spatio-temporal correlations $C$. For calculation of the multifractal characteristics, the length of the temporal window is selected individually for each case to contain an adequate set of statistics (about 1000 events).

The results of calculations of the parameters $C, \alpha_{\min }$ and $S$ for each case presented in Table 1 are shown in Appendix B, (see Figs. B1-B12: b, c, d). The vertical line in each figure marks the corresponding strong earthquake, which is selected for analysis. It is necessary to emphasize, that the presented dynamics corresponds namely to that strong earthquake, which is shown by the vertical line and it does not relate to any other strong earthquake, which one can see in Figs. B1a-B12a. For those earthquakes, another set of statistics have to be selected, and thus the other array of the parameters $C, \alpha_{\min }$ and $S$ have to be obtained. In many cases their statistics are not enough for multifractal analysis, and so we did not consider those earthquakes.

It should be mentioned also, that the variations of multifractal characteristics presented in Figs. B1-B12 appear really due to the processes of the reorganization of the seismicity but not due to numerical errors or instability of the procedure of calculation of generalized fractal dimensions. The example considered in the Appendix C confirms this statement.

Generally, it is seen from Figs. B1-B12 (b, c), that there is a tendency of decreasing of both multifractal characteristics: $\alpha_{\min }$ and entropy $S$ before the mainshocks. It means the increase of spatial inhomogeneity of the seismicity distribution in a wide range of scale levels, preceding strong earthquakes. The seismicity distribution evolves from the more homogeneous (disordered) state towards the more clustered (ordered) state.

The other peculiarity revealed is the enhancement of the level of spatio-temporal correlations in the seismicity before mainshocks (see Figs. B1-B12, d). That indicates the in- 
Table 2. The parameters of the strong earthquakes selected for analysis. $N-$ the number of earthquake, $\varphi-$ latitude of epicenter, $\lambda-$ longitude of epicenter, $z$ - the depth of hypocenter, $M$ - magnitude, $S$ - the square of surrounding area centred on the epicenter of the earthquake

\begin{tabular}{ccccccc}
\hline$N$ & data & $\varphi$ & $\lambda$ & $z, \mathrm{~km}$ & $M$ & $S, \mathrm{~km}^{2}$ \\
\hline 1 & $27 / 10 / 88$ & $36.3^{\circ} \mathrm{N}$ & $141.749^{\circ} \mathrm{E}$ & 40 & 7.7 & $400 \times 400$ \\
2 & $02 / 11 / 89$ & $39.736^{\circ} \mathrm{N}$ & $143.39^{\circ} \mathrm{E}$ & 0 & 6.6 & $400 \times 400$ \\
3 & $07 / 02 / 93$ & $37.658^{\circ} \mathrm{N}$ & $137.309^{\circ} \mathrm{E}$ & 26.5 & 6.6 & $400 \times 400$ \\
4 & $04 / 10 / 94$ & $43.383^{\circ} \mathrm{N}$ & $147.928^{\circ} \mathrm{E}$ & 46.2 & 8.1 & $400 \times 400$ \\
5 & $28 / 12 / 94$ & $40.434^{\circ} \mathrm{N}$ & $143.867^{\circ} \mathrm{E}$ & 14.1 & 7.5 & $400 \times 400$ \\
6 & $07 / 01 / 95$ & $40.232^{\circ} \mathrm{N}$ & $142.425^{\circ} \mathrm{E}$ & 55.9 & 7.2 & $400 \times 400$ \\
7 & $17 / 01 / 95$ & $34.583^{\circ} \mathrm{N}$ & $135.027^{\circ} \mathrm{E}$ & 33.4 & 7.2 & $400 \times 400$ \\
8 & $17 / 02 / 96$ & $37.306^{\circ} \mathrm{N}$ & $142.643^{\circ} \mathrm{E}$ & 59.1 & 6.7 & $400 \times 400$ \\
9 & $19 / 10 / 96$ & $31.798^{\circ} \mathrm{N}$ & $131.972^{\circ} \mathrm{E}$ & 44.6 & 6.6 & $400 \times 400$ \\
10 & $24 / 11 / 87$ & $33.01^{\circ} \mathrm{N}$ & $115.85^{\circ} \mathrm{W}$ & 11.2 & 6.6 & $200 \times 200$ \\
11 & $28 / 06 / 92$ & $34.2^{\circ} \mathrm{N}$ & $116.44^{\circ} \mathrm{W}$ & 1 & 7.3 & $200 \times 200$ \\
12 & $16 / 10 / 99$ & $34.594^{\circ} \mathrm{N}$ & $116.271^{\circ} \mathrm{W}$ & 0 & 7.1 & $200 \times 200$ \\
\hline
\end{tabular}

tensification of formation of spatio-temporal clusters of the seismicity prior to strong earthquake.

The aforementioned tendencies (decrease of the highorder fractal dimension and entropy, and increase of the spatio-temporal correlation) can be clearly seen from Fig. 11 on the example of the well-known Hyogo-ken Nanbu (Kobe) earthquake of 17 January 1995 in Japan $\left(\varphi=34.583^{\circ} \mathrm{N}\right.$, $\lambda=135.02^{\circ} \mathrm{E}, M=7.2$, depth $\left.=33 \mathrm{~km}\right)$. The decrease of the multifractal characteristics $\alpha_{\min }$ and $S$ started approximately two years before the earthquake. After the earthquake, both multifractal characteristics tend to reach their initial level. Such post-earthquake behavior can be considered as a recovery process, when the earthquake focal system breaks down to the more disordered (chaotic) state after release of main portion of seismic energy. As for the level of spatio-temporal correlations $C$, its increase starts approximately three years before the Kobe earthquake. After the earthquake the presence of aftershock sequence cause significant increase of value $C$ due to strong temporal clustering.

In the case of the other earthquakes the seismicity distribution behavior have similar features.

It is necessary to mention that there is no definite scenario of variations of multifractal characteristics of the seismicity spatial distribution prior to the all strong earthquakes. In some cases (see Figs. B1 and B11) the multifractal characteristics decrease rapidly before the earthquake moment. Sometimes the slow trend of decreasing of multifractal characteristics can be seen for periods before the earthquakes (see Figs. B2, B4, B5, and B12). In the case shown on Fig. B8 the multifractal characteristics $S$ and $\alpha_{\min }$ decrease abruptly approximately 2 years before the earthquake. After that the value of $S$ slowly decrease and the value of $\alpha_{\min }$ keeps almost constant value.

In the case of two Japanese earthquakes of 7 January 1995 (Fig. B6) and of 19 October 1996 (Fig. B9), the multifractal characteristics have not any tendency of decreasing before considered strong earthquakes.
Also there is no definite scenario of changes of the level $C$ of spatio-temporal correlations prior to all strong earthquakes considered. So in the cases shown at Figs. B7, B10, B11, and $\mathrm{B} 12$ the value $C$ gradually increase before earthquakes. In the case shown on Fig. B1 the value $C$ increase rapidly before the earthquake moment. In many cases (Figs. B3, B4, B5 and B6) the increase of the level of spatio-temporal correlations $C$ prior to earthquake is not significant in comparison with previous variations of its value. These variations are possibly linked to aftershocks of previous events or earthquake swarms, which are strongly clustered in time.

In the cases shown on Figs. B2, B8 and B9, the level of spatio-temporal correlations $C$ does not increase before the mainshock.

In some cases the decrease of multifractal characteristics or the increase of the value $C$ are not followed by the strong earthquakes. That means that the earthquake clustering occurs not due to crack network development and stress accumulation. The other possible explanation of such a fact could be that the accumulated stress is released by a swarm of earthquakes rather than by one strong earthquake. Anyway, the aforementioned facts reflect the complex nature of seismic process.

In order to reveal the general tendency of variations of characteristics $\alpha_{\min }, S$ and $C$ prior to strong earthquakes, the superposed epoch method is applied to all the earthquakes considered. The dynamics of the values $\alpha_{\min }, S$ and $C$ obtained by this method is shown in Fig. 4. The moment $t=0$ in Fig. 4 corresponds to the considered earthquake moments in Figs. B1-B12.

In this case the length of sliding temporal window for calculation of multifractal characteristics is taken equal to $0.5 \times 10^{8} \mathrm{~s}$ for Japaneese earthquakes and equal to $0.15 \times 10^{8}$ $\mathrm{s}$ for Californian earthquakes. The length of the temporal window for calculation of value $C$ is taken equal to $0.3 \times 10^{8}$ $\mathrm{s}$ for all earthquakes, as previously.

The individual patterns of some earthquakes are neglected 


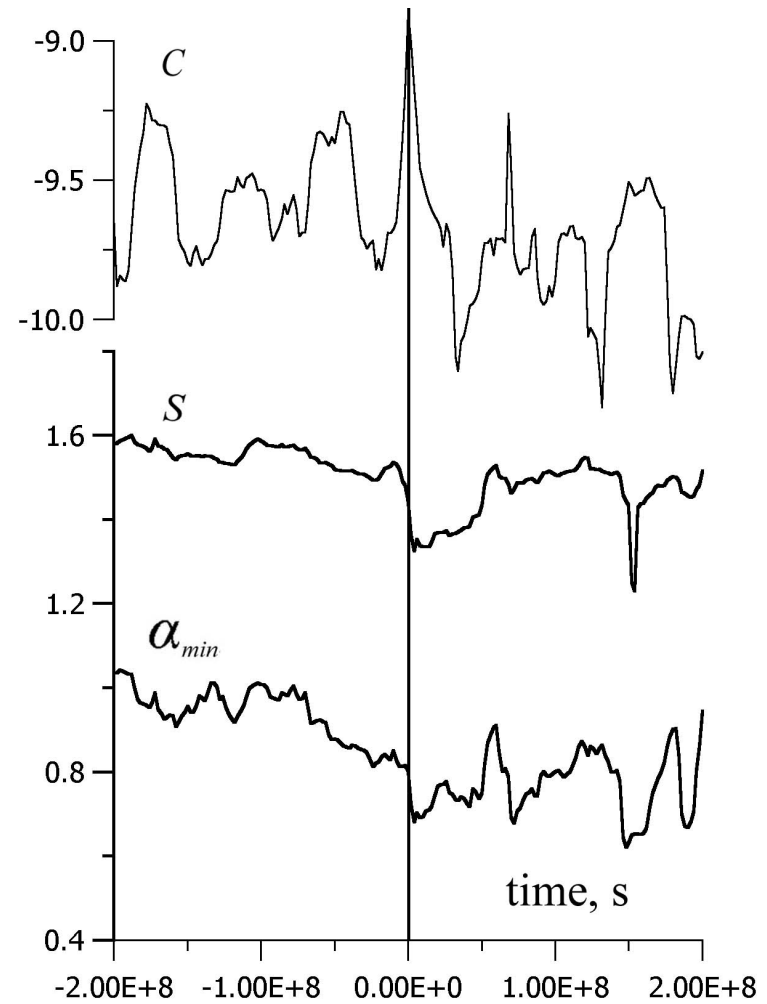

Fig. 4. The superposed epoch analysis results. The curves from top to bottom correspond to variations of the value $C$, the entropy $S$ and the value $\alpha_{\min }$.

in this case, since the length of the temporal windows is not selected individually for each earthquake. But even under such condition the general tendencies can be clearly seen in Fig. 4.

So the multifractal characteristics $\alpha_{\min }$ and $S$ tend to decrease before the strong earthquakes. The value $C$ have a tendency of increase before the strong earthquakes. But in this case some additional peak of average value $C$ appears before the moment when the increase of $C$ is starting. That can be explained by the presence of earthquake swarms, which are strongly clustered in time, for the temporal periods before the strong earthquakes.

Once more we would like to pay attention to the earthquakes with magnitude $M<6.5$ (ex. Fig. B1), which are not preceded by the decrease of multifractal characteristics or increase of the level of spatio-temporal correlations $C$ in the seismicity. The rupture area of these earthquakes is much smaller than the surrounding area selected for analysis. The absence of expected behavior in this case confirms the necessity of taking the size of surrounding area consistent with the size of the rupture area of expected earthquakes in order to reveal precursory signatures.

It should be mentioned also that the methods suggested in this paper may be not suitable for revealing precursory signatures for those strong earthquakes, which are not situated in the central part of the analyzed area. The matter is that if the strong earthquake is occurred at the edge of some area, the big amount of seismicity registered in this area is not related to the preparation process of that earthquake. This feature can be illustrated by the events $\mathrm{N} 1$ and $\mathrm{N} 8$ (see Table 2 and Figs. B1 and B8). There are two earthquakes with magnitude $M>6.5$ on the Figs. B1 and B8, corresponding to these cases. There is no clear tendency of decrease of multifractal characteristics and the increase of the value $C$ before the first earthquake (N 1 in Table 2) with $M>6.5$ on Fig. B8. The area, selected for analysis in this case, is centered in the epicenter of the second strong earthquake (N 8 in Table 2) on this figure. The decrease of multifractal characteristics is observed before this second earthquake. If the center of the area is placed in the epicenter of the first strong earthquake (N 1 in Table 2, Fig. B1), one can see the decrease of multifractal characteristics and increase of the value $C$, which haven't been observed before this earthquake on Fig. B8.

\section{Discussion and conclusions}

The results presented in this paper allow us to conclude that the multifractal characteristics $\alpha_{\min }$ and $S$ tend to decrease and the level of spatio-temporal correlations $C$ tend to increase prior to majority of the considered strong earthquakes. Such tendencies are in good agreement with the results of simulation of the destruction of elastic body with a number of shear cracks (Kiyashchenko and Troyan, 2001).

However, it is difficult to reveal any definite scenario in the behavior of the aforementioned characteristics prior to strong earthquakes. This scenario is individual for each particular case. In some cases the decrease of multifractal characteristics and the increase of the level of spatio-temporal correlations is not followed by the strong earthquake. These facts reflect the complex nature of seismic process.

In few cases the expected behavior of the characteristics of spatio-temporal distribution of the seismicity are not observed prior the earthquake. It is interesting to note that the depth of hypocenters of the earthquakes, for which the decrease of multifractal characteristics (Figs. B6 and B9) or the increase of the level of spatio-temporal correlation are not revealed (Figs. B8 and B9), exceeds $40 \mathrm{~km}$. That allow us to suppose that the absence of the expected behavior of the seismicity (which is well explained in the frame of elastic model) before mainshocks can be related to manifestation of viscous properties of the litosphere material at these depths.

However, in spite of the aforementioned facts, the monitoring of variations of these characteristics is potentially interesting and can be used for seismic hazard risk evaluation together with other precursory signatures.

The effectiveness of methods suggested in this paper will be improved if more complete catalogs with lower magnitude threshold will be available for analysis. The increase of the amount of data will allow to analyze the distribution of hypocenters instead of distribution of epicenters of the seismicity. Moreover, it would allow us to select the surrounding area of the earthquake for analysis as small as necessary, and, therefore, to forecast the earthquakes with lower magni- 
Step 1:

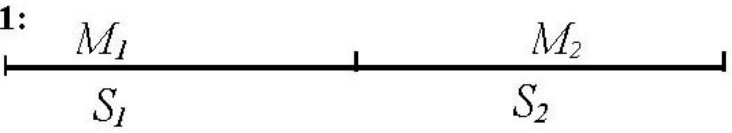

Step 2:

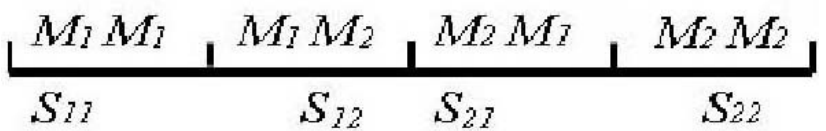

Fig. A1. Multifractal structure generation process.

tude. The elaboration of procedure of filtering of the seismic events, which do not occur due to formation of new rupture area (for example, the dislocation of the rupture sides due to overcoming of friction forces threshold without rupture growth), would make an important contribution to further development of the suggested methods of seismicity distribution analysis.

\section{Appendix A}

Here we give a brief description of main points of multifractal analysis, which helps to understand better the contents of Sect. 2.

Multifractal structure appears as a result of some kind of self-similar multiplicative process. Let us imagine a line (Fig. A1) with normalized mass and length, both equal to unity. Then, at the first step, the line is divided into $K$ equal parts $S_{i}(i=1, \ldots, K)$. In our example (Fig. A1) $K=2$. Then, the mass is distributed along the line in such a way, that the mass of the part with number $i$ is $M_{i}$. The mass of the line is equal to unity, therefore $\sum_{i=1}^{K} M_{i}=1$. At the next step each part $S_{i}$ is also divided into $K$ equal parts $S_{i l}$ $(l=1, \ldots, K)$ and the mass is redistributed along these parts $S_{i}$ in the same proportion as at the first step. Thus the mass of part $S_{i l}$ is $M_{i} M_{l}(l=1, \ldots, \mathrm{K})$. After $n$ steps the line will be divided into $K^{n}$ parts $S_{i_{1} i_{2} \ldots i_{n}}$ with mass $M_{i_{1}} M_{i_{2}} \ldots M_{i_{n}}$ (the indexes $i_{1}, i_{2}, \ldots, i_{n}$ are varying from 1 to $K$ ).

Such multiplicative process with the parameters $M_{1}, M_{2}, \ldots, M_{K}$ generates multifractal distribution of mass along the line. Multifractal functions, generated by binomial $(K=2)$ multiplicative process with the parameters $M_{1}=0.6$ and $M_{2}=0.4$ after 10 steps are shown on Fig. A2.

The left plot on Fig. A2 corresponds to the case when at each step of multiplicative process, the smaller part of mass goes to the right side of each considered segment of line (non-mixed multiplicative process). In the case, which is shown on the right side of Fig. A2, in half of cases the smaller part of mass goes to the left side of the line segment (mixed multiplicative process). Figure A3 demonstrate more inhomogeneous multifractal structures, which have been generated by binomial $(K=2)$ multiplicative process (non-mixed and mixed) with parameters $M_{1}=0.8, M_{2}=0.2$.

In the case of binomial multiplicative process the code $\left(i_{1} i_{2} \ldots i_{n}\right)$ of the part $S_{j}=S_{i_{1} i_{2} \ldots i_{n}}$ of the considered line
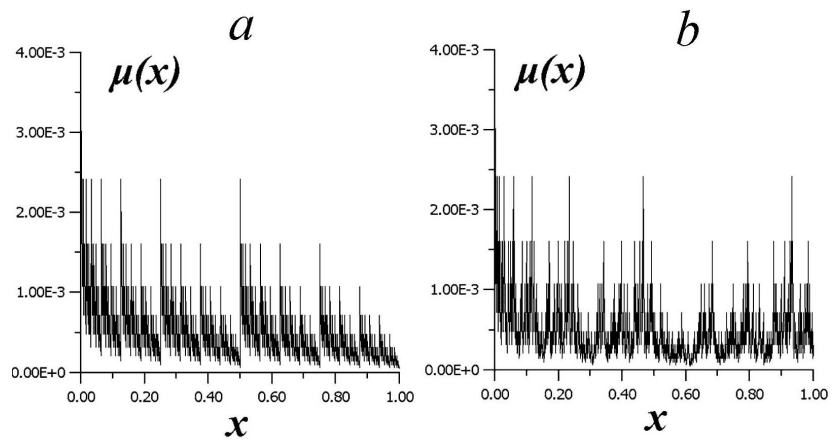

Fig. A2. Multifractal function, generated by binomial multiplicative process with parameters $M_{1}=0.6, M_{2}=0.4$ : (a) non-mixed multiplicative process, (b) mixed multiplicative process.
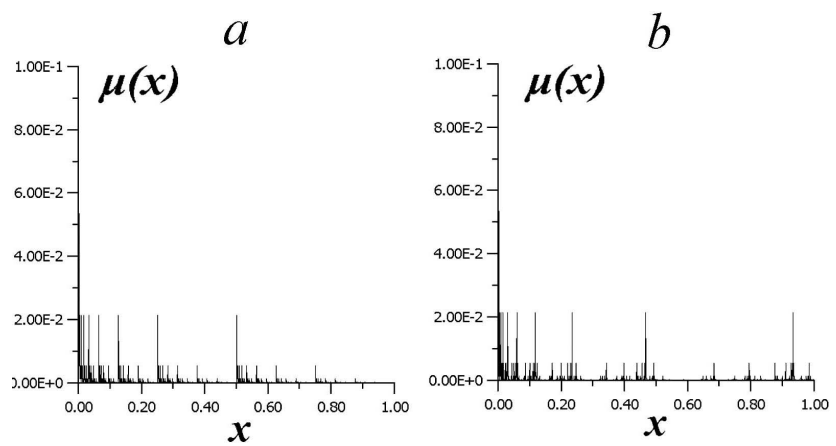

Fig. A3. Multifractal function, generated by binomial multiplicative process with parameters $M_{1}=0.8, M_{2}=0.2$ : (a) non-mixed multiplicative process, (b) mixed multiplicative process.

consist of the numbers " 1 " and " 2 ". If $m$ is the number of the values "1" in the code of the part $S_{j}$, then the mass of this part (the value of multifractal measure function for this part) can be written as follows:

$$
\begin{aligned}
& p_{j}=M_{1}{ }^{m} M_{2}{ }^{n-m}=M_{1}^{n \varphi_{1}} M_{2}^{n \varphi_{2}} ; \\
& \varphi_{1}=\frac{m}{n} ; \varphi_{2}=\frac{n-m}{n}=1-\varphi_{1} .
\end{aligned}
$$

The number of parts of the line with mass $p=p_{j}\left(\varphi_{1}\right)$ can be calculated as:

$N_{n}\left(\varphi_{1}\right)=\frac{n !}{\left(\varphi_{1} n\right) !\left(\varphi_{2} n\right) !}$,

using the well-known formula from combinatorics. According to Stirling formula, $n ! \rightarrow \sqrt{2 \pi} n^{n+1 / 2} e^{-n}$, when $n \rightarrow \infty$. Therefore,

$N_{n}\left(\varphi_{1}\right)=\frac{\exp \left(-n\left(\varphi_{1} \ln \varphi_{1}+\varphi_{2} \ln \varphi_{2}\right)\right)}{\sqrt{2 \pi \varphi_{1} \varphi_{2}}}$.

The size $\Delta$ of the part $S_{j}$ is equal to $2^{-n}$. Then, if $n \rightarrow \infty$, $N_{n}\left(\varphi_{1}\right) \sim g\left(\varphi_{1}\right) \Delta^{-f\left(\varphi_{1}\right)}$.

The Holder exponents $\alpha_{j}$ for each part $S_{j}$ of the line are defined as follows:

$\alpha_{j}=\lim _{\Delta \rightarrow 0} \frac{\ln \left(p_{j}\right)}{\ln (\Delta)} ; \quad p_{j}=\Delta^{\alpha_{j}}$. 


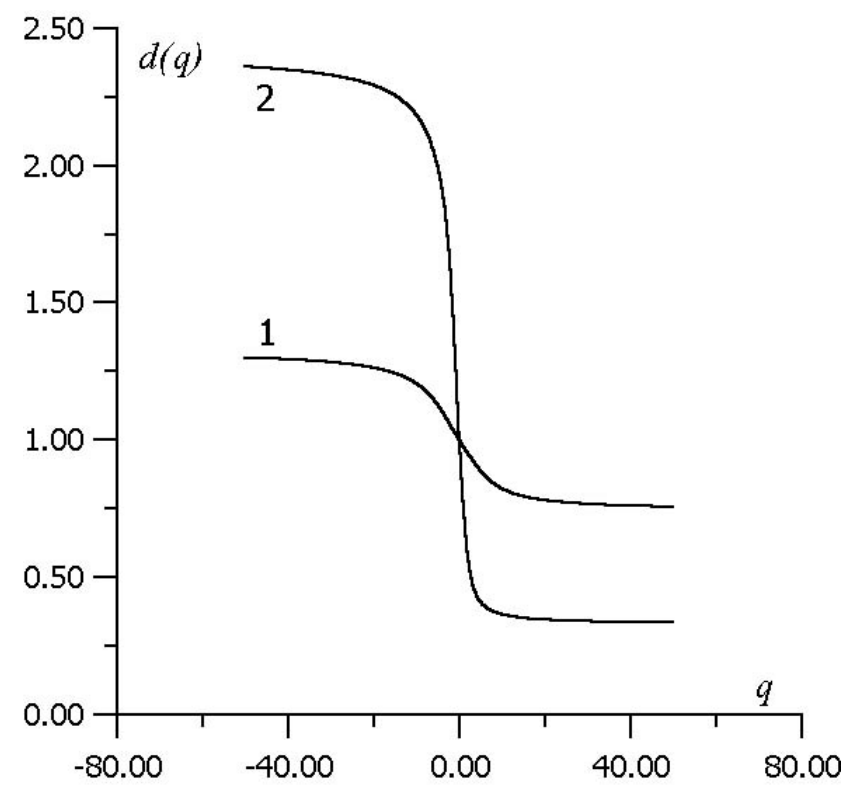

Fig. A4. The spectrums of generalized fractal dimensions $d(q)$ for multifractal functions which are shown on Figs. 7 (curve 1) and 8 (curve 2).

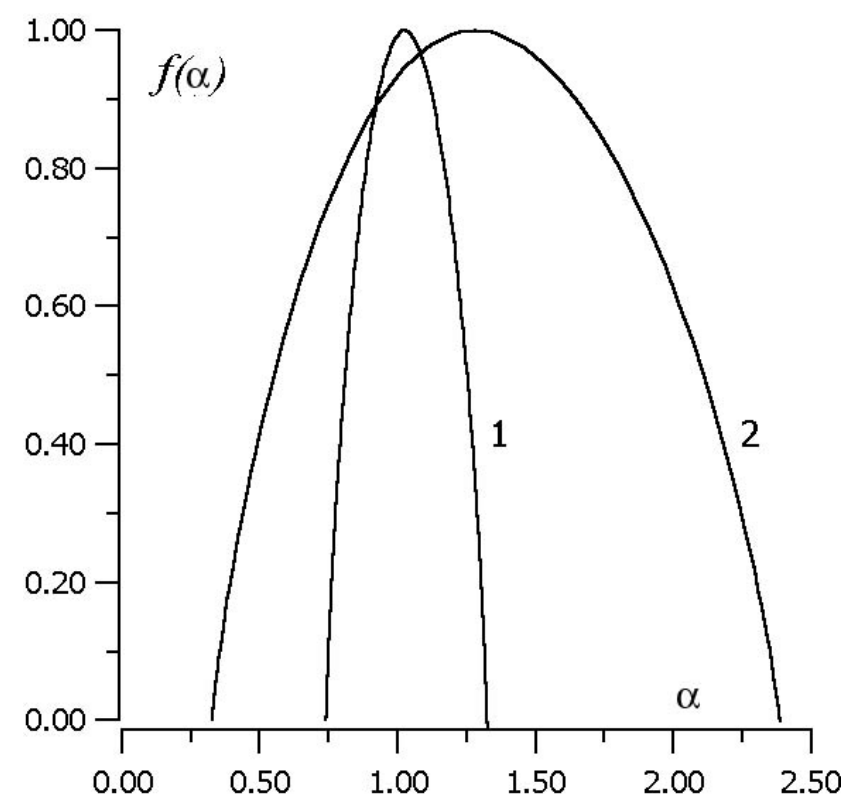

Fig. A5. Multifractal spectrums $f(\alpha)$ for functions which are shown on Figs. 7 (curve 1) and 8 (curve 2).

Then, as $\alpha=\alpha\left(\varphi_{1}\right), N_{n}\left(\varphi_{1}(\alpha)\right) \sim g\left(\varphi_{1}(\alpha)\right) \Delta^{-f\left(\varphi_{1}(\alpha)\right)}$.

Multifractal spectrum $(f(\alpha)$ curve) is defined as:

$f(\alpha)=\lim _{\Delta \rightarrow 0} \frac{\ln \left(N_{n}\left(\varphi_{1}(\alpha)\right)\right.}{\ln (1 / \Delta)}$.

It can be seen that $f(\alpha)$ is the fractal dimension of the subset of segments of line with Holder exponent equal to $\alpha$.

The definition of multifractal spectrum $f(\alpha)$ is one of the ways of description of multifractal structures. Another way is based on calculation of generalized fractal dimensions.

Let us introduce the Renyi entropy of order $q$ :

$\ln \left(I_{q}(\Delta)\right)=\frac{\ln \left(\sum_{j} p_{j}^{q}(\Delta)\right)}{1-q}$, if $q \neq 1$
$\ln \left(I_{q}(\Delta)\right)=-\sum_{i} p_{i}(\Delta) \ln \left(p_{i}(\Delta)\right)$, if $q=1$.

Then, the generalized fractal dimension of the order $q$ is defined as:

$d(q)=\lim _{\Delta \rightarrow 0} \frac{\ln \left(I_{q}(\Delta)\right)}{\ln (1 / \Delta)}$.

The value $d(0)$ is fractal dimension, and $d(2)$ - correlation dimension of the considered mass distribution.

The spectrums of generalized fractal dimensions $d(q)$ for multifractal structures presented on the right sides of Figs. A2 and A3, are shown on Fig. A4 by the curves 1 and 2 , respectively. The difference $w=d(\infty)-d(-\infty)$ is larger for the more inhomogeneous case (Fig. A3).

It is possible to show that the Holder exponent $\alpha$ and $f(\alpha)$ curve can be retrieved from the spectrum of generalized fractal dimensions $d(q)$ using the transforms:

$\alpha(q)=\frac{d}{d q}((q-1) d(q))$

$f(\alpha(q))=q-d(q)(q-1)$.

The multifractal spectrum $f(\alpha)$ contains important information about the parameters of multiplicative process underlying generation of the considered multifractal distribution function generation. The multifactal spectrums calculated for multifractal distributions, which are shown on the right side of Figs. A2 and A3, are demonstrated on Fig. A5 by the curves 1 and 2 , respectively.

The maximal value of $f(\alpha)$ is equal to fractal dimension $d(0)$. The values $\alpha_{\min }$ and $\alpha_{\max }$ are linked correspondingly to maximal $\left(M_{\max }\right)$ and minimal $\left(M_{\min }\right)$ parameters of multiplicative process and to extremal values of spectrum of generalized fractal dimensions:

$\alpha_{\min }=d(\infty)=\frac{1}{\ln K} \ln \left(\frac{1}{M_{\max }}\right) ;$

$\alpha_{\max }=d(-\infty)=\frac{1}{\ln K} \ln \left(\frac{1}{M_{\min }}\right)$.

The value $\alpha_{\min }\left(\alpha_{\max }\right)$ describes the scaling in more densely (sparsely) populated domains of the considered region. It can be seen (Fig. A5) that in the case of distribution with more high contrasts (Fig. A3) the width of multifractal spectrum is larger.

The Holder exponent $S=\alpha_{1}$, satisfying the equation

$\frac{d f\left(\alpha_{1}\right)}{d \alpha_{1}}=1$,

equal to the generalized fractal dimension $d(1)$ and can be expressed as a function of the parameters of multiplicative 


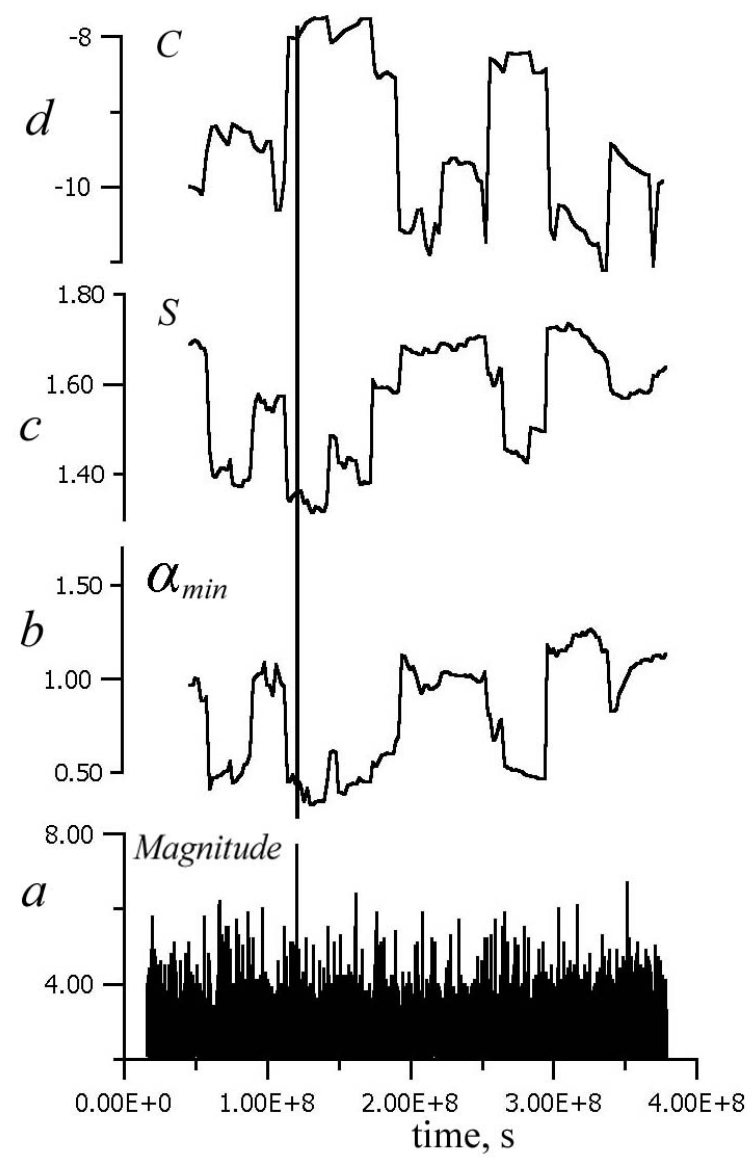

Fig. B1. The results for the earthquake (27 October 1988; Japan; latitude $-36.3^{\circ} \mathrm{N}$; longitude $-141.749^{\circ} \mathrm{E}$; depth $-40 \mathrm{~km}$; Magnitude - 7.7). (a) the magnitudes of the seismic events in the earthquake region; (b), (c), (d) the results of calculation of higherorder generalized fractal dimension $\left(\alpha_{\min }\right)$, entropy $(S)$, and value $C=\ln (g(0,20 \mathrm{~km}))$ correspondingly in the sliding temporal window. The length of temporal window is $0.3 e+08 \mathrm{~s}$ for multifractal characteristics. The square of selected area is $400 * 400 \mathrm{~km}^{2}$.

process:

$S=-\sum_{i=1}^{K} M_{i} \log _{K} M_{i}$

We call it the entropy of multifractal measure generation process since it has a form of informational entropy. The values of entropy $S$ for the multifractal spectrums, which are calculated for distribution functions shown on Figs. A2 and A3, are correspondingly $S=0.98$ and $S=0.73$. It is seen that the entropy $S$ is smaller for more inhomogeneous distribution case.

\section{Appendix B}

Here we present the results of calculations of the values of multifractal characteristics $\alpha_{\min }, S$ and the level of spatiotemporal correlations $C$. Calculations are performed in slid-

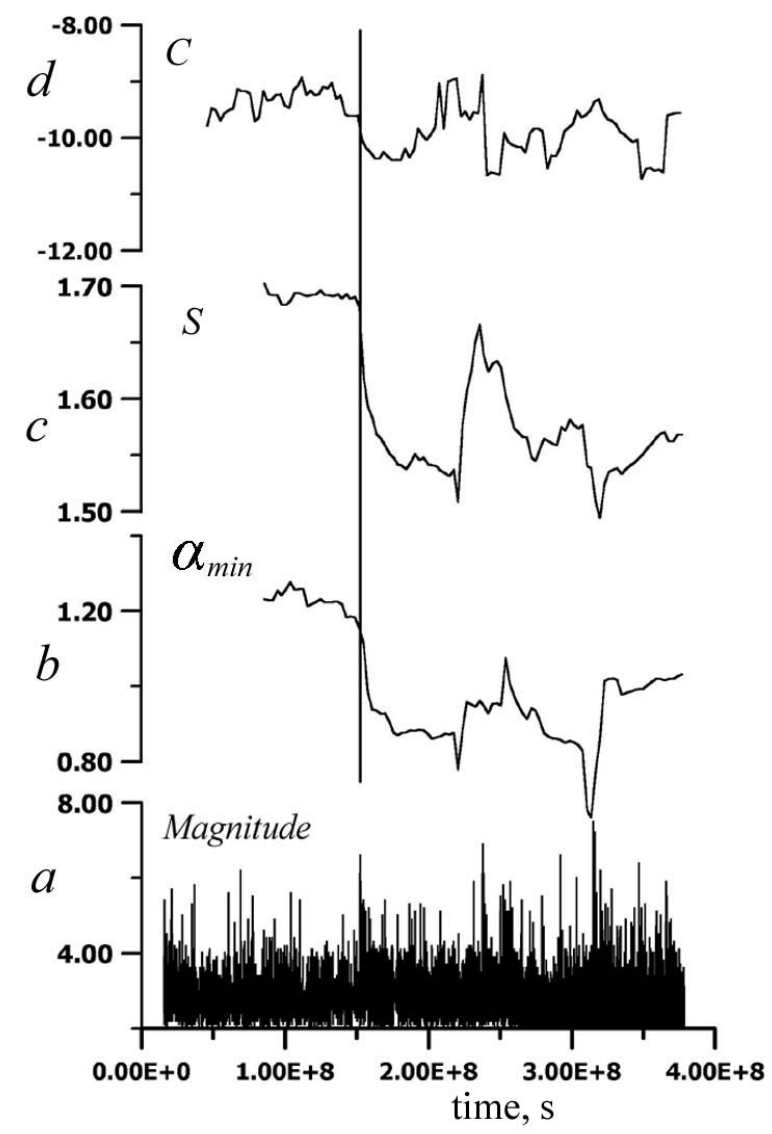

Fig. B2. The results for the earthquake (2 November 1989; Japan; latitude $-39.736^{\circ} \mathrm{N}$; longitude $-143.39^{\circ} \mathrm{E}$; depth $-0 \mathrm{~km}$; Magnitude - 6.6). (a) the magnitudes of the seismic events in the earthquake region; (b), (c), (d) the results of calculation of higherorder generalized fractal dimension $\left(\alpha_{\min }\right)$, entropy $(S)$, and value $C=\ln (g(0,20 \mathrm{~km}))$ correspondingly in the sliding temporal window. The length of temporal window is $0.7 e+08 \mathrm{~s}$ for multifractal characteristics. The square of the selected area is $400 * 400 \mathrm{~km}^{2}$.

ing temporal window for all considered earthquakes. The length of temporal window was taken equal to $0.3 \times 10^{8} \mathrm{~s}$ in the case of calculation of the value $C$ and was selected individually for each earthquake in the case of calculation of multifractal characteristics (see Sect. 3). The results of calculations are shown on Figs. B1-B12 (corresponding to earthquakes $N 1-12$ in Table 1). On each figure (B1 to B12) the main earthquake, for which the seismicity in the surrounding area has been studied, is indicated by the vertical line. The subplot (a) show the magnitudes of seismic events occurred in the earthquake region. The subplots (b), (c), (d) show the results of calculations of higher order fractal dimension $\left(\alpha_{\min }\right)$, entropy $(S)$ and the value $C=\ln (g(0,20 \mathrm{~km}))$ correspondingly in sliding temporal window. 


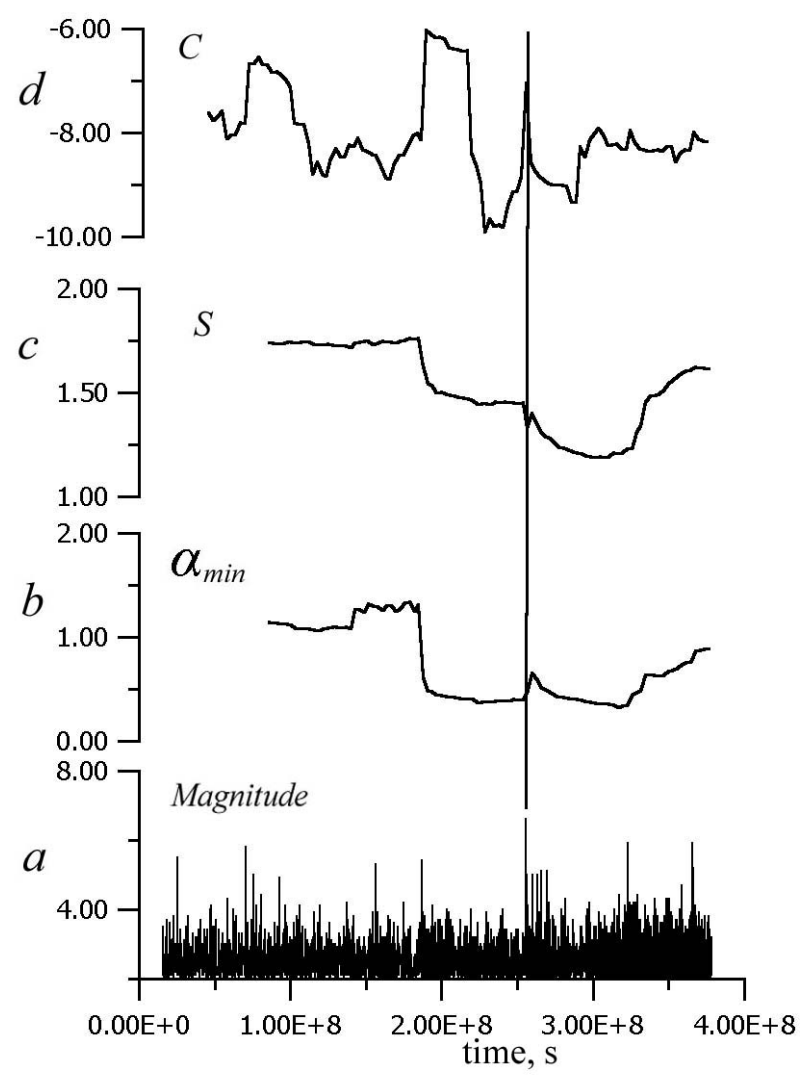

Fig. B3. The results for the earthquake (7 February 1993; Japan; latitude $-37.658^{\circ} \mathrm{N}$; longitude $-137.309^{\circ} \mathrm{E}$; depth $-26.5 \mathrm{~km}$; Magnitude - 6.6). (a) the magnitudes of the seismic events in the earthquake region; (b), (c), (d) the results of calculation of higher-order generalized fractal dimension $\left(\alpha_{\min }\right)$, information entropy $(S)$, and value $C=\ln (g(0,20 \mathrm{~km}))$ correspondingly in the sliding temporal window. The length of temporal window is $0.6 e+08 \mathrm{~s}$ for multifractal characteristics. The square of selected area is $400 * 400 \mathrm{~km}^{2}$.

\section{Appendix C}

In this part an example of calculation of the multifractal characteristics $S$ and $\alpha_{\min }$ in sliding temporal window for the synthetic seismicity catalog with randomly distributed epicenters is presented in order to verify the significance of the variations of multifractal characteristics for the case of real seismicity.

The subcatalog of seismicity registered in the surrounding area of the earthquake $\mathrm{N} 1$ (see Table 2) is taken for test calculation. The coordinates of the epicenters of the earthquakes in this subcatalog were randomly changed. As the result of such procedure, the synthetic seismicity catalog with randomly distributed epicenters is obtained.

The dynamics of characteristics $S$ and $\alpha_{\min }$, which are calculated by sliding temporal window of $0.5 \times 10^{8}$ for synthetic catalog, is shown on Fig. C1 by upper curves. Lower curves on this figure represent the results of calculation of the same characteristics (in the same temporal window) for the real

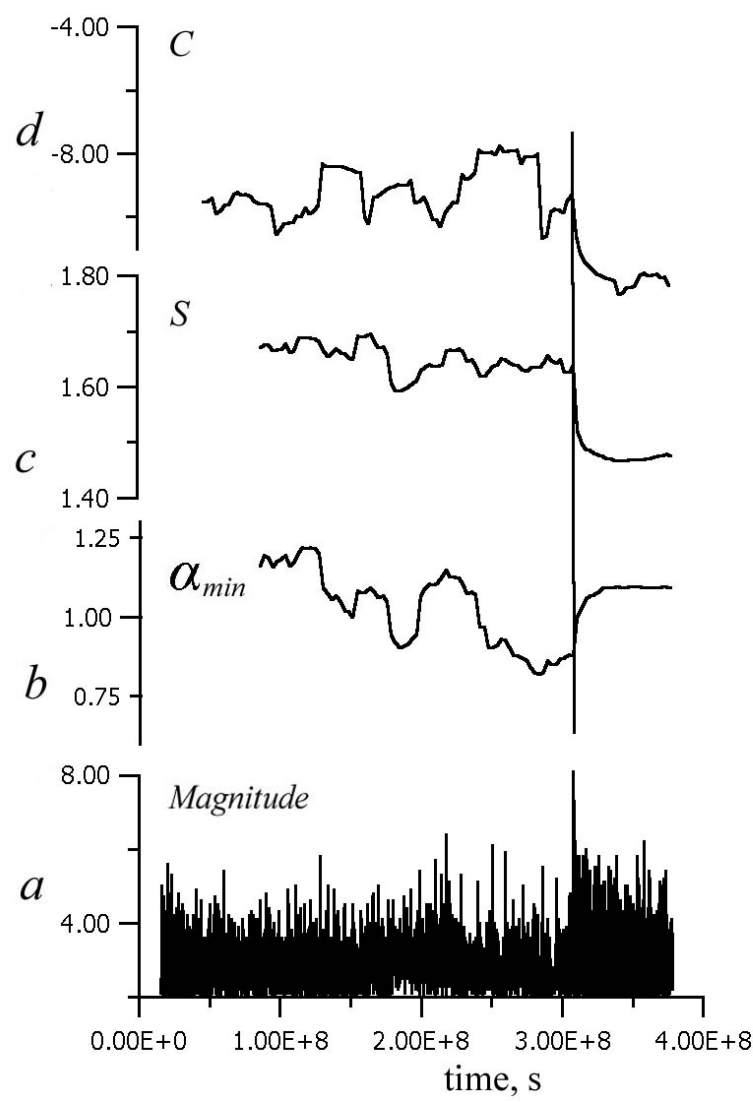

Fig. B4. The results for the earthquake (4 October 1994; Japan; latitude $-43.383^{\circ} \mathrm{N}$; longitude $-147.928^{\circ} \mathrm{E}$; depth $-46.2 \mathrm{~km}$; Magnitude - 8.1). (a) the magnitudes of the seismic events in the earthquake region; (b), (c), (d) the results of calculation of higher-order generalized fractal dimension $\left(\alpha_{\min }\right)$, information entropy $(S)$, and value $C=\ln (g(0,20 \mathrm{~km}))$ correspondingly in the sliding temporal window. The length of temporal window is $0.6 e+08 \mathrm{~s}$ for multifractal characteristics. The square of the selected area is $400 * 400 \mathrm{~km}^{2}$.

seismicity catalog (related to the earthquake $\mathrm{N} 1$ ).

One can see that the lower values of multifractal characteristics of the real seismicity indicate stronger spatial clustering in comparison with those obtained for artificial (randomized) seismicity.

The variations of the values of $S$ and $\alpha_{\min }$ are considerably larger for the case of real seismicity. Moreover, the variations obtained for real seismicity are not correlated with variations obtained for artificial seismicity, which are mainly determined by the number of earthquakes in the temporal window.

Therefore, the variations of multifractal characteristics of real seismicity are significant and not related to instability of the procedure of their estimation or dependence of their estimation on the number of earthquakes in the temporal window. These variations reflect the real processes of seismicity reorganization in wide range of scales.

Acknowledgement. This work has been supported by the grant RFBR 02-05-65081 of Russian Foundation for Basic Research, Pro- 


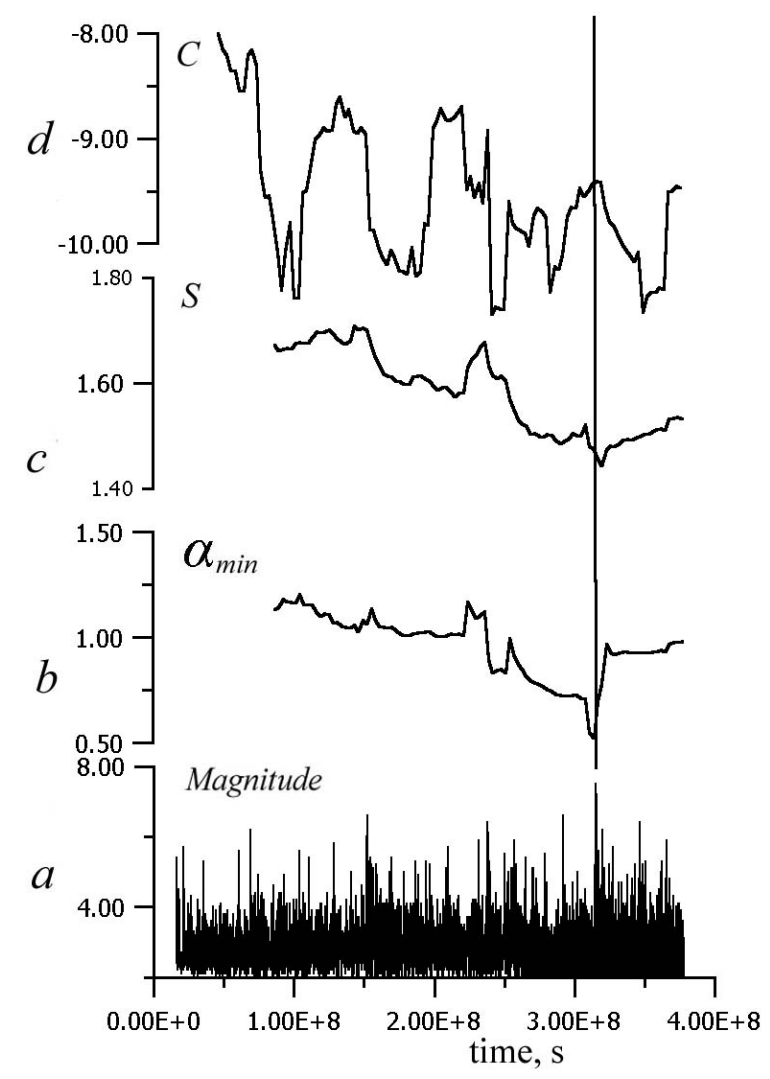

Fig. B5. The results for the earthquake (28 December 1994; Japan; latitude $-40.434^{\circ} \mathrm{N}$; longitude $-143.867^{\circ} \mathrm{E}$; depth $-14.1 \mathrm{~km}$; Magnitude - 7.5). (a) the magnitudes of the seismic events in the earthquake region; (b), (c), (d) the results of calculation of higherorder generalized fractal dimension $\left(\alpha_{\min }\right)$, entropy $(S)$, and value $C=\ln (g(0,20 \mathrm{~km}))$ correspondingly in the sliding temporal window. The length of temporal window is $0.7 e+08 \mathrm{~s}$ for multifractal characteristics. The square of the selected area is $400 * 400 \mathrm{~km}^{2}$.

gramme "Intergeophysica" of Russian Ministry of Higher Education, and grant INTAS 99-1102.

\section{References}

Feder, J.: Fractals, Plenum Press, New York, 1988.

Geilikman, M. B., Golubeva, T. V., and Pisarenko, V. F.: Multifractal patterns of seismicity, Earth. Plan. Sci. Lett., 99, 127-133, 1990.

Hauksson, E.: Crustal structure and seismicity distribution adjacent to the Pacific and North America plate boundary in Southern California, J. Geophys. Res., 105, 13, 875-903, 2000.

Hirata, T. and Imoto, M.: Multifractal analysis of spatial distribution of microearthquakes in the Kanto region, Geophys. J. Let., 107, 155-162, 1991.

Hirata, T., Satoh, T., and Ito, K.: Fractal structure of spatial distribution of microfracturing in rock, Geophys. J. R. Astr. Soc., 90, 369-374, 1987.

Kiyashchenko, D. and Troyan, V.: The peculiarities of shear crack pre-rupture evolution and distribution of seismicity before strong earthquakes, Nat. Haz. Earth Sys. Sc., 1, 3, 145-158, 2001.

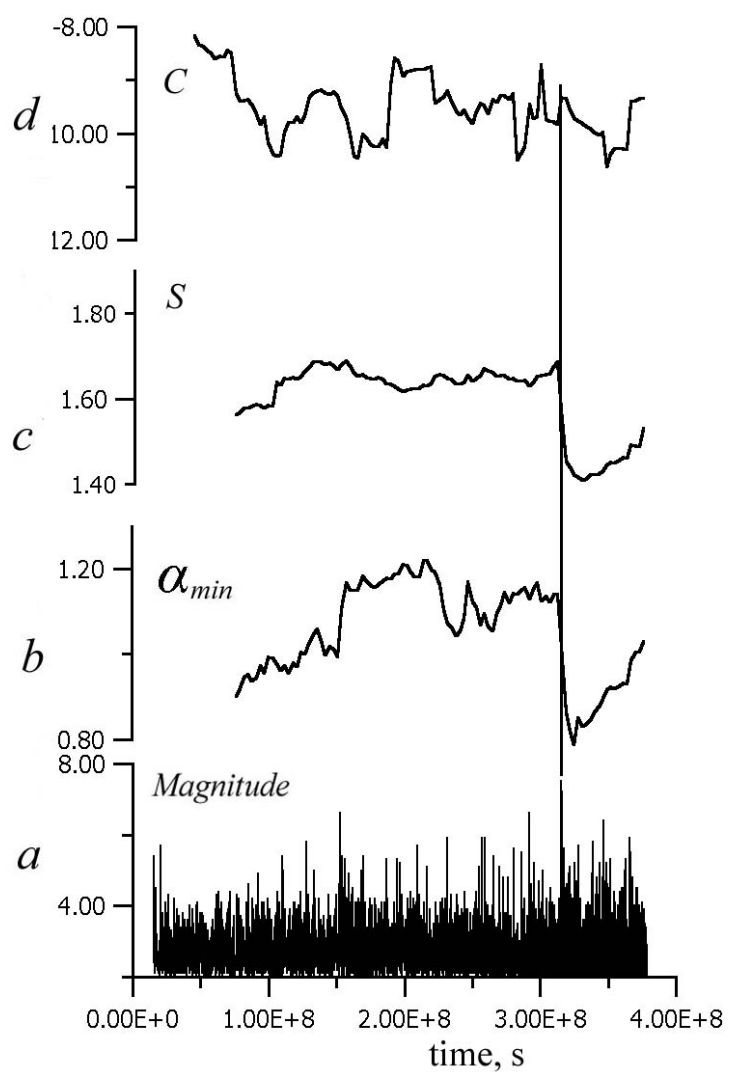

Fig. B6. The results for the earthquake (7 January 1995; Japan; latitude $-40.232^{\circ} \mathrm{N}$; longitude $-142.425^{\circ} \mathrm{E}$; depth $-55.9 \mathrm{~km}$; Magnitude - 7.2). (a) the magnitudes of the seismic events in the earthquake region; (b), (c), (d) the results of calculation of higherorder generalized fractal dimension $\left(\alpha_{\min }\right)$, entropy $(S)$, and value $C=\ln (g(0,20 \mathrm{~km}))$ correspondingly in the sliding temporal window. The length of temporal window is $0.6 e+08 \mathrm{~s}$ for multifractal characteristics. The square of the selected area is $400 * 400 \mathrm{~km}^{2}$.

Lapenna, V., Macchiato, M., Piscitelli, S., and Telesca, L.: Scaleinvariance Properties in Seismicity of Southern Apennine Chain (Italy), Pure Appl. Geophys., 157, 589-601, 2000.

Lawn, B. R. and Wilshaw, T. R.: Fracture of brittle solids, Cambridge, Cambridge University Press, 1977.

Liebowits, H.: Fracture, an advanced treatise, Academic Press, New York, 2, 1968.

Mandelbrot, B.: Multifractal Measures, Especially for the Geophysicist, PAGEOPH, 131, 6-41, 1989.

Mogi, K.: Source locations of elastic shocks in the fracturing process in rocks., Bull. Earthquake. Res. Inst., Univ. Tokio, 46, 1103-1125, 1968;

Okubo, P. G. and Aki, K.: Fractal geometry in the San Andreas fault system, J. Geophys. Res., 92, 345-355, 1987.

Sadovsky, M. , Golubeva, T., Pisarenko, V., and Shnirman, M.: Characteristic dimensions of rocks and hierarchical properties of the seismicity, Isv. Acad. Sci. USSR Phys. Solid Earth, Engl. transl., 20, 87-96, 1984.

Scholz, C.: Experimental study of the fracturing process in the brittle rock, J. Geophys. Res., 73, 1447-1454, 1968.

Shebalin, P., Zaliapin, I., and Keilis-Borok, V.: Premonitory raise of 


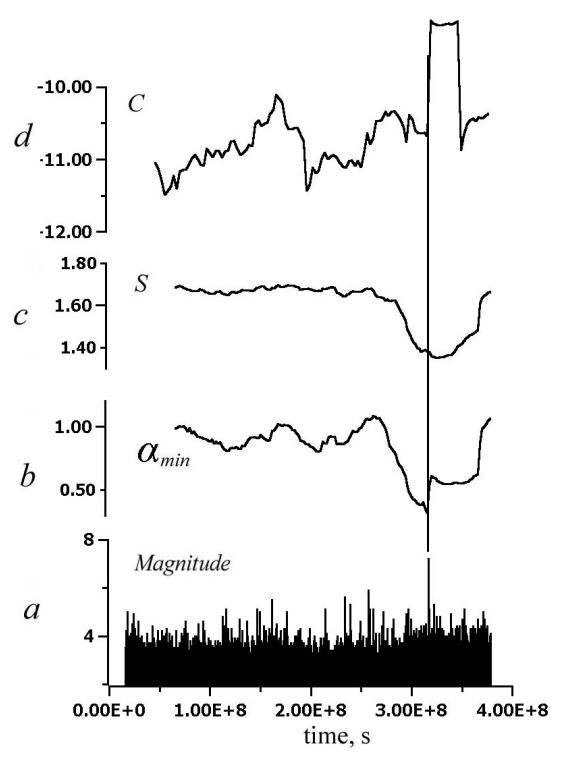

Fig. B7. Results for the earthquake $N 7$ (Kobe earthquake) (Japan; 17 Jan. 1995; lat. $-34.583^{\circ} \mathrm{N}$; long. $-135.027^{\circ} \mathrm{E}$; depth-33.4 km; Mag.-7.2). (a) the magnitudes of the seismic events in Kobe region; (b), (c), (d) the results of calculation of higher-order generalized fractal dimension $\left(\alpha_{\min }\right)$, entropy $(S)$, and value $C=$ $\ln (g(0,20 \mathrm{~km}))$ correspondingly in the sliding temporal window. The length of temporal window is $0.5 e+08 \mathrm{~s}$ for multifractal characteristics.

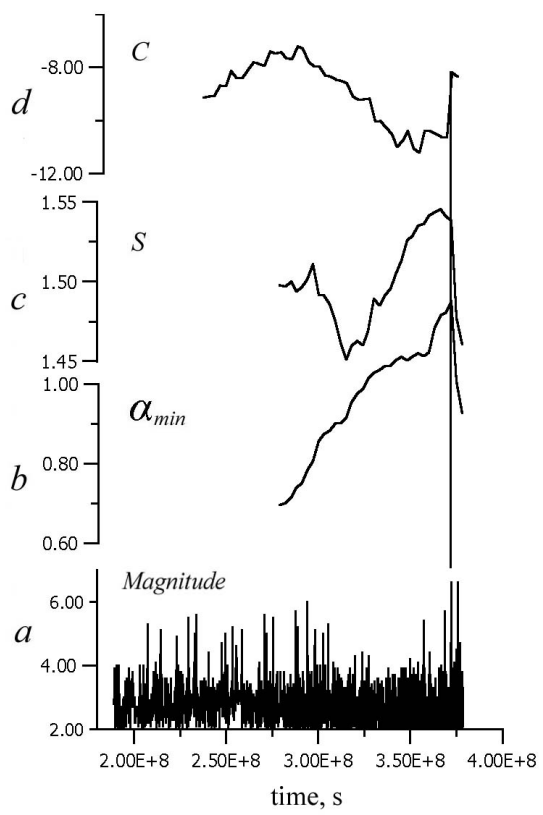

Fig. B9. Results for the earthquake (19 Oct. 1996; Japan; lat.$31.798^{\circ} \mathrm{N}$; long. $-131.972^{\circ} \mathrm{E}$; depth-44.6 km; Mag.-6.6). (a) the magnitudes of the seismic events in the earthquake region; (b), (c), (d) the results of calculation of higher-order generalized fractal dimension $\left(\alpha_{\min }\right)$, entropy $(S)$, and value $C=\ln (g(0,20 \mathrm{~km}))$ correspondingly in the sliding temporal window. The length of temporal window is $0.9 e+08 \mathrm{~s}$ for multifractal characteristics.

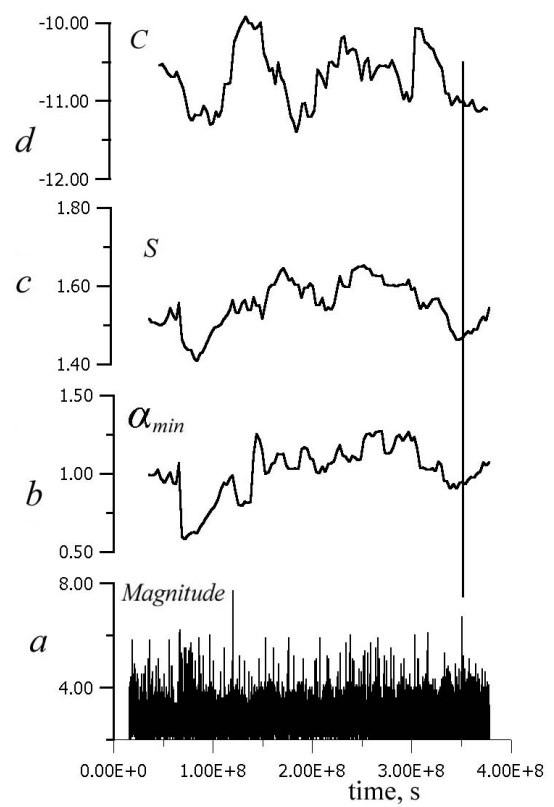

Fig. B8. Results for the earthquake (17 Feb. 1996; Japan; lat.$37.306^{\circ} \mathrm{N}$; long. $-142.643^{\circ} \mathrm{E}$; depth-59.1 km; Mag.-6.7). (a) the magnitudes of the seismic events in the earthquake region; (b), (c), (d) the results of calculation of higher-order generalized fractal dimension $\left(\alpha_{\min }\right)$, entropy $(S)$, and value $C=\ln (g(0,20 \mathrm{~km}))$ correspondingly in the sliding temporal window. The length of temporal window is $0.2 e+08 \mathrm{~s}$ for multifractal characteristics.

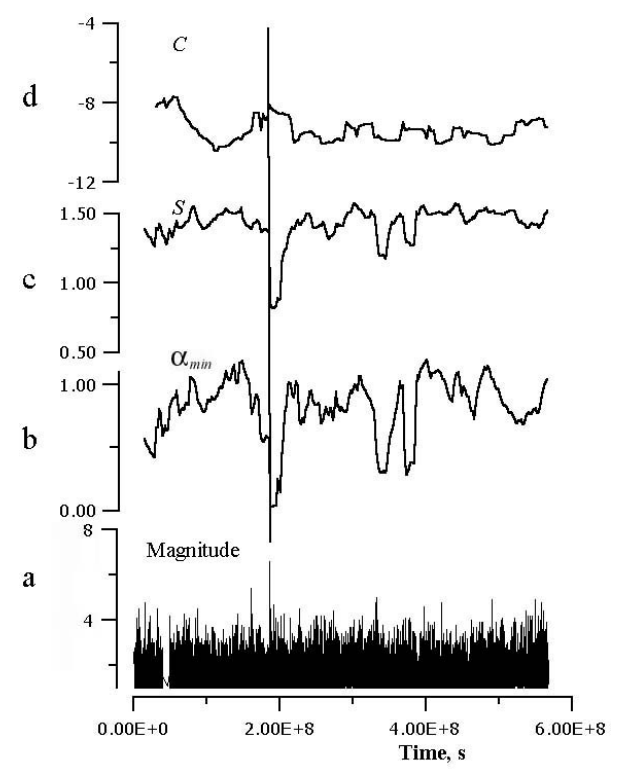

Fig. B10. Results for the earthquake (24 Nov. 1987; Southern California; lat. $-33.01^{\circ} \mathrm{N}$; long. $-115.85^{\circ} \mathrm{W}$; depth-11.2 km; Mag.6.6). (a) the magnitudes of the seismic events in the earthquake region; (b), (c), (d) the results of calculation of higher-order generalized fractal dimension $\left(\alpha_{\min }\right)$, entropy $(S)$, and value $C=$ $\ln (g(0,20 \mathrm{~km}))$ correspondingly in the sliding temporal window. The length of temporal window is $0.15 e+08 \mathrm{~s}$ for multifractal characteristics. 


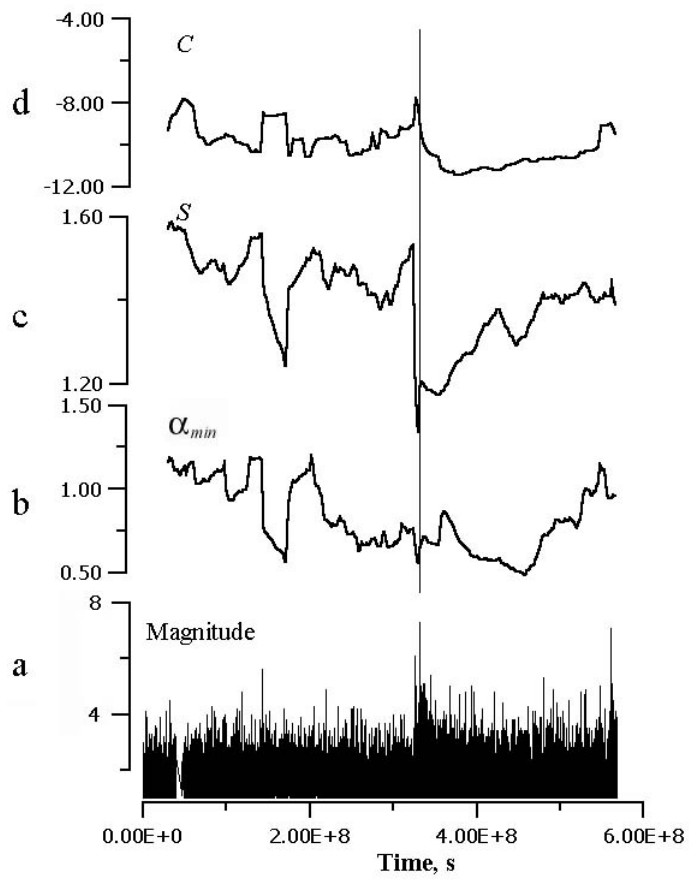

Fig. B11. The results for the earthquake (28 June 1992; Southern California; latitude $-34.2^{\circ} \mathrm{N}$; longitude $-116.44^{\circ} \mathrm{W}$; depth $-1 \mathrm{~km}$; Magnitude - 7.3). (a) the magnitudes of the seismic events in the earthquake region; (b), (c), (d) the results of calculation of higherorder generalized fractal dimension $\left(\alpha_{\min }\right)$, entropy $(S)$, and value $C=\ln (g(0,20 \mathrm{~km}))$ correspondingly in the sliding temporal window. The length of temporal window is $0.15 e+08 \mathrm{~s}$ for multifractal characteristics. The square of the selected area is $200 * 200 \mathrm{~km}^{2}$.

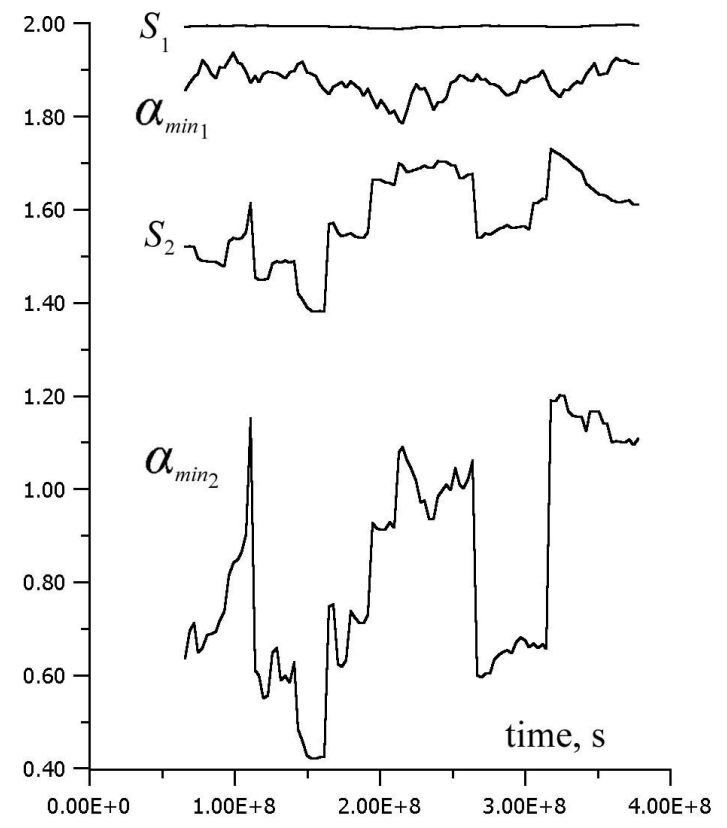

Fig. C1. The results of calculation of multifractal characteristics $S$ and $\alpha_{\min }$ by sliding temporal window with the length $0.5 e+08 \mathrm{~s}$ for the synthetic (upper curves) and real (lower curves) seismicity catalogs.

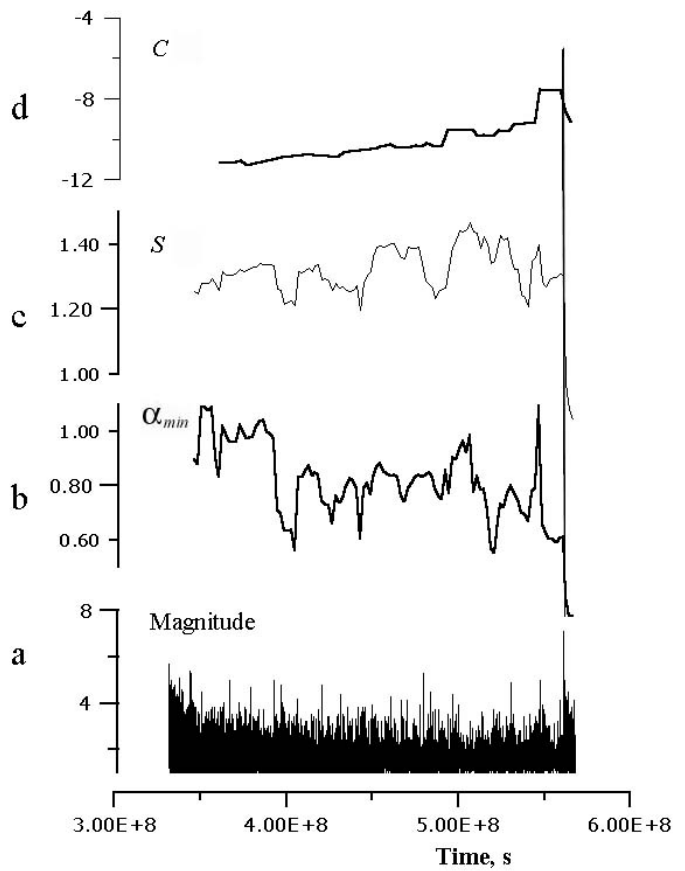

Fig. B12. The results for the earthquake (16 October 1999; Southern California; latitude $-34.594^{\circ} \mathrm{N}$; longitude $-116.271^{\circ} \mathrm{W}$; depth $-0 \mathrm{~km}$; Magnitude -7.1). (a) the magnitudes of the seismic events in the earthquake region; (b), (c), (d) the results of calculation of higher-order generalized fractal dimension $\left(\alpha_{\min }\right)$, entropy $(S)$, and value $C=\ln (g(0,20 \mathrm{~km}))$ correspondingly in the sliding temporal window. The length of temporal window is $0.15 e+08 \mathrm{~s}$ for multifractal characteristics. The square of the selected area is $200 * 200 \mathrm{~km}^{2}$. 
the earthquakes correlation range: Lesser Antilles, Phys. Earth Plan. Int., 122, 241-249, 2000.

Sobolev, G.: Fundamentals of earthquake forecasting, "Nauka", Moscow, 1990.

Sobolev, G. and Ponomarev, A.: Rock fracture stages from acoustic emission patterns in the laboratory experiment, Volcanology and Seismology, 4-5, 50-62, 1999.

Turcotte, D. L.: Fractal tectonics and erosion, Fractals, 1, 3, 491504, 1997.
Uritsky, V. and Troyan, V.: Estimation of changes in fractal geometry of distributed seismicity during periods of major earthquakes, in: Problems of Geophysics, Is. 35, SPbU Press, 39-42, 1998.

Wang, J.-H. and Lee, C.-W.: Multifractal measures of earthquakes in west Taiwan, PAGEOPH, 146, 131-145, 1996.

Zavialov, A. and Nikitin, Y.: Process of seismicity localisation before strong Kamchatka earthquakes, Volcanology and Seismology, 4-5, 83-89, 1999. 